Original Research Paper

\title{
Some Basic Reactions in Nuclear Fusion
}

\author{
${ }^{1}$ Relly Victoria Virgil Petrescu, ${ }^{2}$ Aversa Raffaella, ${ }^{3}$ Samuel Kozaitis, \\ ${ }^{2}$ Apicella Antonio and ${ }^{1}$ Florian Ion Tiberiu Petrescu

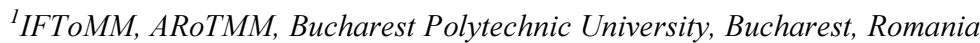 \\ ${ }^{2}$ Advanced Material Lab, Department of Architecture and Industrial Design, \\ Second University of Naples, 81031 Aversa (CE) Italy \\ ${ }^{3}$ Florida Institute of Technology, USA
}

Article history

Received: 06-07-2017

Revised: $13-07-2017$

Accepted: 19-07-2017

Corresponding Author: Florian Ion Tiberiu Petrescu IFToMM, ARoTMM, Bucharest Polytechnic University, Bucharest, Romania Email: scipub02@gmail.com

\begin{abstract}
Over time it has advanced the idea that the achievement of a hot nuclear reaction can require tens or hundreds of millions of degrees. Precise calculations clearly indicate a much higher temperature. At least 10 million degrees are necessary for $1 \mathrm{keV}$ in thermonuclear reaction. At $400 \mathrm{keV}$ it needs a temperature of 4000 million degrees to occur the hot fusion reaction. Hot fusion needs a temperature of about 4000 million degrees, or 4 billion degrees if we believe in the calculations the radius of deuterium static. If we believe in the calculations the radius of the real, dynamic deuterium, in movement, the temperature required to achieve the warm fusion reaction increases still 10000 times, reaching a value of 40 trillions degrees. Unfortunately, this clarification does not bring us closer to the realization of the hot fusion reaction, but on the contrary, us away from the day when we will be able to achieve it. Today we have only made 150 million degrees. A huge problem is even the achievement of such temperatures. For these reasons we are entitled to think up next following, namely achieving the cold fusion. Authors propose to bomb the fuel with accelerated Deuterium nuclei.
\end{abstract}

Keywords: Nuclear Fusion, Cold Nuclear Fusion, Thermonuclear Reaction, Possible Nuclear Reactions, Nuclear Power, Some Fusion Solutions, Fusion, Heavy Water, Hydrogen Nuclei, Deuterium, Tritium, Lithium, Helium, Nuclear Energy Forces, Velocities, Powers, Dimensions

\section{Introduction}

Nuclear fusion is the process by which two or more atomic nuclei join, or "fuse", to form a single core more difficult. During this process, the raw material is not preserved because some of the mass of the fusing nuclei is converted into energy which is released (Shultis and Faw, 2002; Progress in Fusion, ITER; Moses et al., 2009; Kramer, 2011; Atzeni and Meyer-ter-Vehn, 2004).

The energy from the connection of the kernel is greater than the energy from the connection of each of the cores of the melted which for the production.

Fusion is the process by which one powers the stars active (Hoffman, 2013; Park et al., 2005; Jones, 1986; Miller and Krakowski, 1984; Schewe and Stein, 2005).

There are many experiments which will examine the possibility of the energy generated by merger plan for the generation of electricity. Nuclear fusion has a high potential as a source of sustainable energy. This is due to the abundance of hydrogen on the planet and the nature of inert helium (the core which would result from the nuclear fusion of atoms of hydrogen; Hydrogen, from Wikipedia).

Unfortunately, the reaction of a nuclear fusion controlled has not yet been carried out by the people, due to the temperatures required to support one (Gerstner, 2009; Palmer, 1997).

Some of the merger may be used in the design of the atomic weapons, although it is generally for fission and not by the merger, which is associated with the achievement of atomic bomb. Is worthy of notice of the fact that the merger may also have a role to play in the design of the bomb with hydrogen (Thermonuclear weapon, from Wikipedia).

The fusion of the two cores with smaller than those of iron (which, together with nickel has the highest 
energy on the tying nucleon) issue, in general, energy, while the fusion of nuclei heavier than iron absorbs energy. The opposite is true for the reverse process of, nuclear fission. This means that the merger takes place, in general, for items easier only and also that normally, fission (Vermote et al., 2008) takes place only for items more heavy.

There is no upcoming events extreme astrophysics which may lead to short periods of merger with more cores heavy. It is a process that gives rise to nucleosynthesis, the creation of heavy elements, during some events as well as supernovae (Reynolds et al., 2008).

Create the necessary conditions for the merger on the ground is very difficult, up to the point that has not been carried out at any scale for Protium, common isotope of hydrogen, suffering from the natural merger into stars.

To nuclear weapons, a part of the energy released by an atomic bomb (fission bomb) is used for compression and heating of a fuel of merger containing radionuclide heavier than hydrogen and also sometimes, lithium, up to the point of "ignition". At this time, the energy released in the reactions of fusion is sufficient to maintain on the short reaction. Nuclear experiments based on merger attempts to create similar conditions, using means much smaller with all the present these experiments have not managed to maintain the necessary conditions for the illumination of the long enough for the merger to be a source of commercial energy viable.

The origin of the energy released in fusion of the light elements is due to the interaction of two opposing forces: Nuclear force (Brown and Rechenberg, 1996), which attracts together protons and neutrons and the force Coulomb (1788) which causes protons to reject each other. Protons are positive charge and will repel each other, but they still stick between them, showing the existence of other forces mentioned as a point of attraction, force powerful nuclear which exceed the electrical repulsion within a very close range. The effect of this force is not observed outside the nucleus. Therefore, the force has a powerful dependency on distance, which makes it a force of short-range. The same force pulls also the neutrons together or neutrons and protons together. Because the nuclear force is stronger than the force of the Coulomb for atomic nuclei smaller than of iron and nickel, building these cores of the cores easier, by means of the quantities of extra energy of merger resulting from the attraction of the net weight of these particles (Krane, 1987).

For the cores the larger, however, not power (energy) is released because the nuclear force is the short-range and may not continue to act beyond the atomic nuclei still larger. Thus, the energy is no longer released when these cores are made by merger (in exchange, energy is absorbed in such processes; Ragheb, 2013).
The reactions to the merger of the light elements give the energy of the stars and produce virtually all the elements by a process of nuclear material, called nucleosynthesis. The merger of the lightest in the stars releases the power (and the mass that accompanies it always). For example, in the fusion of two nuclei of hydrogen to form helium, seventenths of $1 \%$ of the weight is carried by the system in the form of the kinetic energy or other forms of energy (such as electromagnetic radiation). Anyway, the elements heavier than iron production may only to absorb energy.

Research in the field of fusion controlled with the aim to produce energy by merger for the production of electricity, has been carried out for over 60 years. She was accompanied by difficulties and the scientific and technological extreme, but had as a result progress. At present, the reactions of merger controlled have been unable to produce (self sustaining) the reaction of the merger controlled.

There is a need for considerable energy to force the cores to merge, even those of the more easily element of hydrogen. This is due to the fact that all cores have a positive charge (because of their protons) and how they will repel each other, the cores are the objection to a strong resistance when trying to their proximity or to be placed too close together. Accelerated at high speeds (being heated at temperatures of huge thermonuclear fusion; or accelerated in circular accelerators), they may exceed this electrostatic repulsion helping nuclear force attractive to be strong enough to obtain the merger (Petrescu, 2012; Petrescu and Calautit, 2016a; De Ninno et al., 2002).

Fusion of cores easier, which creates a nucleus more difficult and many times a neutron free or a proton, in general, releases more energy than necessary to force the cores together; this is a trial exothermically, which can cause reactions of self-support (De Ninno et al., 2002).

The energy released in most nuclear reactions is much higher than in the chemical reaction, as the energy which binds the cores (or nucleons) together is much higher than the energy that retains the electrons of a kernel. For example, the energy (the ionization energy) obtained by adding an electron to a core of hydrogen is $13.6 \mathrm{eV}$, less than one millionth of the $17 \mathrm{MeV}$ released in one reaction between Deuterium-Tritium (D-T), (Shultis and Faw, 2002).

The reactions of merger have a density of energy many times greater than nuclear fission; the reactions of fusion energy produce much higher for each unit of mass than the reactions of individual fission, which are themselves by millions of times more energetic than the chemical reactions. Only the total conversion (direct conversion) of mass into energy (such as that caused by the annihilation process of collision between matter and 
antimatter) is more energetic per unit of mass than the nuclear fusion (Petrescu and Petrescu, 2012).

When a nucleon, such as a proton or a neutron (Reid, 1968) is added to a kernel, nuclear force shall draw it to the other nucleons, but especially to its neighbors immediately, because of the short range of the force. Nucleons from the inside of the nucleus have several neighboring nucleons than those on the surface of the core.

As the smaller nuclei have a larger area in relation to the ratio area-volum, the nucleon binding energy due to the nuclear force which is increased in general with the size of the nucleus, but he went up to a limit value corresponding to that of a core having a diameter of approximately four nucleons.

It is important to take into account the fact that the above description is a model of the toy, because nucleons are objects of quantum mechanics (Tipler and Llewellyn, 2008) and so on, for example, since two neutrons from a core are identical between them, by making a distinction from one another (such as which one is in the interior and which is located on the surface) is actually meaningless sense and the inclusion of the quantum mechanics is necessary for the real calculations.

On the other hand the force electrostatic is a square force inverted, so a proton which is added to a nucleus shall support a repulsive electrostatic force from the other all protons in the nucleus.

The energy electrostatic per nucleon due to the force electrostatic thus increases without limit when the nuclei become larger.

The next few decades are of crucial importance for the implementation of a strategy to reduce greenhouse gas emissions.

By the end of the century, given the growth in population, the increase in urbanization and the expansion of the electricity grid in developing countries, energy demand will have tripled.

The use of fossil fuels that shaped industrial civilization is synonymous with emissions of greenhouse gases and pollution.

There is an urgent need to find a large-scale, non- $\mathrm{CO}_{2}$ emitting, sustainable and available source of energy.

The merger offers the following advantages:

\section{$\mathrm{No} \mathrm{CO}_{2}$ Emissions}

Fusion does not generate carbon dioxide or other greenhouse gases. The main by-product is helium, a nontoxic inert gas.

\section{No Risk of Core Meltdown}

A Fukushima nuclear accident can't occur in a fusion reactor. Conditions conducive to fusion reactions are difficult to achieve; In the event of disturbance, the plasma cools within a few seconds and the reactions cease. Moreover, the quantity of fuel present in the enclosure is insufficient to feed the reactions beyond a few seconds and a "chain reaction" is inconceivable from the point of view of physics.

\section{Abundant Energy}

At the same mass, the fusion of light atoms releases an energy nearly four million times greater than that of a chemical reaction such as coal, oil or gas and four times that of nuclear fission reactions.

Fusion can provide the basic energy needed to meet the electricity needs of our cities and industries.

\section{Sustainability}

Fuel fusion is universally available and virtually inexhaustible.

Deuterium can be obtained from water; the tritium will be produced during the fusion reaction when the neutrons from the fusion of the nuclei interact with the lithium of the modules placed in the vacuum chamber (Lithium reserves in the earth's crust would permit the operation of melting plants for more than 1,000 years and those of the oceans could meet the needs for millions of years).

\section{No Long-Lived High-Level Radioactive Waste}

Nuclear fusion reactors do not produce high-level, long-lived radioactive waste. The activation of the components of a fusion reactor is sufficiently low that the materials can be recycled or reused within 100 years of the plant being shut down.

\section{No Proliferation}

Fusion does not use fissile materials such as uranium and plutonium (radioactive tritium is not a fissile or fissionable material). A fusion reactor contains no elements that can be used to manufacture nuclear weapons.

\section{Cost}

The amount of energy produced by an industrial fusion reactor, as it will be produced in the second half of this century, will be equivalent to that produced by a fission reactor - between 1 and 1.7 GigaWatts.

The average cost per kilowatt of electricity should also be of the same order: Slightly higher at the beginning, the technology being new, then decreasing thereafter as economies of scale reduce prices (Avantages de la Fusion, ITER).

The energy mix of the coming decades will have to rely on a wide variety of different energy sources. As a new energy option that does not emit carbon and does not produce long-lived high-level nuclear waste, fusion meets the challenges of maintaining major climate balances, resource availability and safety. 


\section{Materials and Methods}

First time Deuterium was extracted from water in 1931 by Harold Urey (Harold Urey, from Wikipedia). Small linear electrostatic accelerators have indicated that D-D reaction (fusion of two deuterium nuclei) is exothermic, even at that time.

It is known that not only the second isotope of hydrogen (Deuterium) can produce fusion nuclear energy, but and the third (heavy) isotope of hydrogen (Tritium) may produce energy through a nuclear fusion.

First nuclear fusion reaction it is possible between two nuclei of Deuterium and may be obtained: One Tritium nucleus plus a proton and energy, either a helium isotope with a neutron and energy (Equation 1 and 2; Petrescu, 2012; Petrescu and Calautit, 2016a; Petrescu and Petrescu, 2012; Petrescu et al., 2016):

$$
\begin{aligned}
& { }_{1}^{2} \mathrm{D}+{ }_{1}^{2} \mathrm{D} \rightarrow{ }_{1}^{3} \mathrm{~T}+1.01 \mathrm{MeV}+{ }_{1}^{1} \mathrm{H}+3.02 \mathrm{MeV} \\
& ={ }_{1}^{3} \mathrm{~T}+{ }_{1}^{1} \mathrm{H}+4.03 \mathrm{MeV} \\
& { }_{1}^{2} \mathrm{D}+{ }_{1}^{2} \mathrm{D} \rightarrow{ }_{2}^{3} \mathrm{He}+0.82 \mathrm{MeV}+{ }^{1} n+2.45 \mathrm{MeV} \\
& ={ }_{2}^{3} \mathrm{He}+{ }^{1} n+3.27 \mathrm{MeV}
\end{aligned}
$$

It is known that a Deuterium nucleus has a proton and a neutron while a Tritium nucleus has a proton and two neutrons. Today we know that fusion reaction may occur and between a nucleus of Deuterium and one of Tritium (Equation 3) and this fusion nuclear reaction may be produced more easily than one between two deuterons (Equation 1 and 2):

$$
\begin{aligned}
& { }_{1}^{2} \mathrm{D}+{ }_{1}^{3} \mathrm{~T} \rightarrow{ }_{2}^{4} \mathrm{He}+3.5 \mathrm{MeV}+{ }^{1} n+14.1 \mathrm{MeV} \\
& ={ }_{2}^{4} \mathrm{He}+{ }^{1} n+17.6 \mathrm{MeV}
\end{aligned}
$$

As can be seen in the Equation 3, not only that the reaction between Tritium and Deuterium it is much easier to be carried out, but it is also more generous, donating approx. 5 times more energy (Petrescu, 2012; Petrescu and Calautit, 2016a; Petrescu and Petrescu, 2012; Petrescu et al., 2016).

Another important nuclear reaction which can give much energy may be produced between a nucleus of Deuterium and an isotope of Helium (Equation 4):

$$
\begin{aligned}
& { }_{1}^{2} \mathrm{D}+{ }_{2}^{3} \mathrm{He} \rightarrow{ }_{2}^{4} \mathrm{He}+3.6 \mathrm{MeV}+{ }_{1}^{1} \mathrm{H}+14.7 \mathrm{MeV} \\
& ={ }_{2}^{4} \mathrm{He}+{ }_{1}^{1} \mathrm{H}+18.3 \mathrm{MeV}
\end{aligned}
$$

The isotope of helium ${ }_{2}^{3} \mathrm{He}$ is obtained in the reaction of 2 and it together with Deuterium may produce the reaction of the 4 only if the Deuterium has enough power (energy). This is a final reaction which produces much energy and a helium atom ${ }_{2}^{4} \mathrm{He}$ which is a non toxic inert gas, very stable.

The reaction of the 1 generates Tritium which together with Deuterium (if one of them or both have enough energy) produce the nuclear reaction of 3 to generate a lot of energy and helium.

As basic we have the final nuclear reactions 3 and 4, which produce a lot of power (energy) and helium, a non toxic, inert and very stable gas. For this reason the group of nuclear reactions of fusion is an advantageous one, friendly, pure, peacefully and inexpensive.

Naturally the Tritium appears in the nuclear reactor, only when the reaction of 1 is produced. But because the reaction of the 3 is the most desired, can use a simple trick to produce this reaction more often, simply by multiplying the quantity of Tritium from nuclear reactor by a supporting reaction (Equation 5):

${ }_{3}^{6} \mathrm{Li}+{ }^{1} n \rightarrow{ }_{1}^{3} \mathrm{~T}+{ }_{2}^{4} \mathrm{He}+4.784 \mathrm{MeV}$

The bars of lithium are easily entered or extracted in the nuclear reactor and by this mechanism can be controlled very simple and the fusion reaction speed at any time.

When we want to accelerate the fusion reaction one introduces more bars of lithium simultaneously. When it wants to diminish the speed of nuclear reaction one must introduce less bars of lithium, or more out of them. If it is desired to stop the nuclear reaction of merger instantly it needs just get out all the bars of lithium from the inside of the reactor shortly.

The reaction of the 5 can help much the reaction to the merger, by controlling its production.

Lithium reserves in the earth's crust would permit the operation of melting plants for more than 1,000 years and those of the oceans could meet the needs for millions of years.

Neutrons necessary for producing the reaction 5 are generated even in the reactor in the framework of the reactions 2 and 3 .

The reaction of the supporting 5, enter the lithium and produces the demand for Tritium, the additional energy and the same inert gas, helium (Petrescu, 2012; Petrescu and Calautit, 2016a; Petrescu and Petrescu, 2012; Petrescu et al., 2016).

Raw materials to achieve nuclear fusion are the deuterium and lithium, more exactly heavy water and bars of lithium. Result a lot of energy and helium.

The reaction can be controlled easily through various methods. The reaction of the merger no tends to "get out of control" such as to the fission (being difficult to start it and easy to stop it).

An extremely important equation to achieve nuclear fusion on earth is between two atoms (or ions) of tritium (Equation 6). 
Equation 6 can generates extra energy and is much easier to achieve than other possibilities:

$$
{ }_{1}^{3} \mathrm{~T}+{ }_{1}^{3} \mathrm{~T} \rightarrow{ }_{2}^{4} \mathrm{He}+2 \cdot{ }^{1} \mathrm{n}+11.3 \mathrm{MeV}
$$

In a nuclear reactor of this type, supplied with Deuterium, can take place and other nuclear reactions, of which the most important are (Equation 7-9):

$$
\begin{aligned}
& { }_{2}^{3} \mathrm{He}+{ }_{2}^{3} \mathrm{He} \rightarrow{ }_{2}^{4} \mathrm{He}+2 \cdot{ }_{1}^{1} \mathrm{H}+12.9 \mathrm{MeV} \\
& { }_{2}^{3} \mathrm{He}+{ }_{1}^{3} \mathrm{~T} \rightarrow{ }_{2}^{4} \mathrm{He}+{ }_{1}^{1} \mathrm{H}+{ }^{1} \mathrm{n}+12.1 \mathrm{MeV} \\
& { }_{2}^{3} \mathrm{He}+{ }_{1}^{3} \mathrm{~T} \rightarrow{ }_{2}^{4} \mathrm{He}+4.8 \mathrm{MeV}+{ }_{1}^{2} \mathrm{D}+9.5 \mathrm{MeV} \\
& ={ }_{2}^{4} \mathrm{He}+{ }_{1}^{2} \mathrm{D}+14.3 \mathrm{MeV}
\end{aligned}
$$

Lithium with deuterium can still generate four other important reactions (Equation 10-13):

$$
\begin{aligned}
& { }_{1}^{2} \mathrm{D}+{ }_{3}^{6} \mathrm{Li} \rightarrow 2 \cdot{ }_{2}^{4} \mathrm{He}+22.4 \mathrm{MeV} \\
& { }_{1}^{2} \mathrm{D}+{ }_{3}^{6} \mathrm{Li} \rightarrow{ }_{2}^{3} \mathrm{He}+{ }_{2}^{4} \mathrm{He}+{ }^{1} n+2.56 \mathrm{MeV} \\
& { }_{1}^{2} \mathrm{D}+{ }_{3}^{6} \mathrm{Li} \rightarrow{ }_{3}^{7} \mathrm{Li}+{ }_{1}^{1} \mathrm{H}+5.0 \mathrm{MeV} \\
& { }_{1}^{2} \mathrm{D}+{ }_{3}^{6} \mathrm{Li} \rightarrow{ }_{4}^{7} \mathrm{Be}+{ }^{1} \mathrm{n}+3.4 \mathrm{MeV}
\end{aligned}
$$

Lithium can react and with hydrogen (a proton; Equation 14) or with an isotope of $\mathrm{He}$ (Equation 15):

$$
\begin{aligned}
& { }_{1}^{1} \mathrm{H}+{ }_{3}^{6} \mathrm{Li} \rightarrow{ }_{2}^{4} \mathrm{He}+1.7 \mathrm{MeV}+{ }_{2}^{3} \mathrm{He}+2.3 \mathrm{MeV} \\
& ={ }_{2}^{4} \mathrm{He}+{ }_{2}^{3} \mathrm{He}+4.0 \mathrm{MeV} \\
& { }_{2}^{3} \mathrm{He}+{ }_{3}^{6} \mathrm{Li} \rightarrow 2 \cdot{ }_{2}^{4} \mathrm{He}+{ }_{1}^{1} \mathrm{H}+16.9 \mathrm{MeV}
\end{aligned}
$$

These reactions are good because they can enhance the energy produced in the reactor.

It should also be mentioned separately an extremely exciting nuclear reaction (equation 16) that may occur between the stable isotope of boron with 6 neutrons (boron has five protons) and an atom (or ion = proton) of hydrogen.

The reaction between hydrogen and boron can be achieved more easily than others and can generate a large amount of energy plus the inert gas, $\mathrm{He}$ :

$$
{ }_{1}^{1} \mathrm{H}+{ }_{5}^{11} \mathrm{~B} \rightarrow 3 \cdot{ }_{2}^{4} \mathrm{He}+8.7 \mathrm{MeV}
$$

Such a reaction (Equation 16) should be treated completely separate, with no direct connection with the fusion of deuterium or tritium.
But it can also be used together with the reactions already mentioned, to add more energy in reactor to startup of the other fusion reactions. Boron is very much like silicon under the rule similarity diagonally.

Non metal boron has electrical conductivity, type semiconductor. Boron, combining with carbon yields a substance harder than diamond, which is used in the manufacture of special steels (Ferro boron) and of different alloys with tungsten, copper and chromium (Petrescu, 2012; Petrescu and Calautit, 2016a; Petrescu and Petrescu, 2012; Petrescu et al., 2016). Because not found naturally pure, it can be produced industrially by chemical reactions or by reducing chlorine boron in a hydrogen atmosphere, using an electric arc between $1200-1400^{\circ} \mathrm{C}$. Its energy of fusion is about $50.2 \mathrm{~kJ} / \mathrm{mol}$.

\section{Results}

Without fusion, there would be no life on Earth.

What we perceive in the form of light and heat is the result of fusion reactions occurring in the heart of the Sun and the stars. During this process, hydrogen nuclei collide and fuse to give rise to heavier helium atoms and considerable amounts of energy (Halliday and Robert, 1966).

The gravitational force of stars creates the necessary conditions for fusion. Billions of years ago, the hydrogen clouds of the primitive Universe gathered under the effect of gravity and gave rise to very massive stellar bodies. Their extremely dense and warm core is the seat of the Universe fusion process. All these processes which are lighting (Petrescu and Calautit, 2016b) and warming the Universe are processes of warm fusion, thermonuclear reactions.

To produce such a thermonuclear reaction on earth, some special conditions are needed. First the required temperatures should reach some values hard to imagine today and much hard to achieve them.

It has long been believed that the temperature required to heat the merger would be about tens or hundreds of millions of degrees.

In some articles (Petrescu, 2012; Petrescu and Petrescu, 2012) it was shown that the thermonuclear fusion necessary temperature is about 4 billion degrees. The general theory was then redesigned, cataloging the required temperature to start thermonuclear fusion to about 1.2-4 billion degrees. Unfortunately the paper's authors already mentioned (Petrescu, 2012; Petrescu and Petrescu, 2012) had considered (at the first step) the radius of a proton, or deuteron known, calculated or measured approximately, static. Reversing the calculations and determining a deuteron radius (with a high precision) in movement (Petrescu and Calautit, 2016a; 2012b; Petrescu et al., 2016), using genuine relationships, it has established a new temperature required to startup the thermonuclear reaction, about 
10,000 times greater than the temperature initial considered in 2011-2012 (Petrescu, 2012; Petrescu and Petrescu, 2012). Instead of about 4 billion degrees, we know now that is necessary for start the thermonuclear reaction a temperature of about 40 trillion degrees. This is not a temperature easily achieved and maintained, so the first thought takes us to the reaction of cold fusion.

\section{Discussion}

In cold fusion, it is needed to accelerate deuterons (Deuterium ions) and then to be bumped each other.

First one should determine the necessary velocity of the accelerated particles needed to start cold fusion when they will collide. It may use the original Equation 17 (Petrescu and Calautit, 2016a; Petrescu et al., 2016).

This speed has the value: $v=691664.8602[\mathrm{~m} / \mathrm{s}]$.

Second one could determine the radius $\mathrm{R}$ of a moving Deuterium particle, using the relationship 18 (Petrescu and Calautit, 2016a; Petrescu et al., 2016).

Third one may calculate the potential energy of the two adjacent Deuterium particles on fusion (Petrescu, 2012; Halliday and Robert, 1966). This is the minimum translational kinetic energy that must reach a Deuterium particle, accelerated, to produce fusion by collision (using the form 19):

$$
\begin{aligned}
& \left\{\begin{array}{l}
x^{4}+a x^{3}+b x^{2}-c x+d=0 \\
a=2 c^{2} \\
b=\frac{c^{2}\left(q_{1} q_{2}\right)^{2}}{5 h^{2} \varepsilon_{0}^{2}} \\
c=\frac{2 c^{4}\left(q_{1} q_{2}\right)^{2}}{5 h^{2} \varepsilon_{0}^{2}} \\
d=\frac{c^{4}\left(q_{1} q_{2}\right)^{4}}{100 h^{4} \varepsilon_{0}^{4}}
\end{array}\right. \\
& R=\sqrt{\frac{5}{4}} \cdot \frac{h \cdot \sqrt{c^{2}-v^{2}} \cdot \sqrt{c^{2}-\frac{v^{2}}{2}-c \cdot \sqrt{c^{2}-v^{2}}}}{\pi \cdot m_{0} \cdot c^{2} \cdot v} \\
& E_{p}=\frac{q_{1} \cdot q_{2}}{8 \pi \cdot \varepsilon_{0} \cdot R}
\end{aligned}
$$

With $m_{0}$ deuteron $=3.34524 \mathrm{E}-27[\mathrm{~kg}]$ and $v=$ $691664.8602[\mathrm{~m} / \mathrm{s}]$, the radius of one Deuteron at this velocity (calculated with 18 ) takes the below value:

- $\quad R_{D d}=1.91788 \mathrm{E}-19[\mathrm{~m}]($ dynamic at $v=0.002307088 \mathrm{c})$

- Static (with classical calculations) $R_{D s}=1.827 \mathrm{E}-$ $15[\mathrm{~m}]$
Potential energy has the below value (for a dynamic radius, in movement; Equation 20):

$$
\begin{aligned}
& U=E p=6.01333 E-10[\mathrm{~J}]=3753521838[\mathrm{eV}] \\
& =3753521.838[\mathrm{KeV}]=3753.521838[\mathrm{MeV}] \\
& =3.753521838[\mathrm{GeV}]
\end{aligned}
$$

Potential energy has the below value (for a static radius; Equation 21):

$$
U=E p=6.31 E-14[J]=3.94 E 05[\mathrm{eV}]=394[\mathrm{KeV}]
$$

This means that according to the static calculations required temperature of fusion to warm is about 4 billion degrees (Petrescu, 2012).

But calculating using radius determined dynamically, when the energy required is much higher (Equation 20), we come to a temperature necessary for the attainment of the thermonuclear fusion reaction of 40 trillion degrees, as for any $1 \mathrm{keV}$ is needed about 10 million degrees temperature (Petrescu and Calautit, 2016a; Petrescu et al., 2016).

To increase naturally the Brownian motion of particles, for any one $\mathrm{keV}$ it is necessary more 10 million degrees.

Neither the partisans of thermonuclear fusion should not be disappointed or disarmed, because today the merger by both methods can be started and performed by using some tricks. E.g., particle acceleration can be used and in hot merger.

Lithium reactions can greatly help starting and even maintaining the reaction. The paper has also proposed another helper reaction, which also may be tested in the future: That between boron and hydrogen.

\section{Conclusion}

Controlled fusion reaction is now much closer to achievement.

The presented paper proposes first to determine the necessary velocity of the accelerated particles needed to start cold fusion when they will collide. It may use the original Equation 17.

This speed has the value: $v=691664.8602[\mathrm{~m} / \mathrm{s}]$. Second one could determine the radius $\mathrm{R}$ of a moving Deuterium particle, using the relationship 18. Third one may calculate the potential energy of the two adjacent Deuterium particles on fusion.

This is the minimum translational kinetic energy that must reach a Deuterium particle, accelerated, to produce fusion by collision (using the form 19).

Today the merger by both methods can be started and performed by using some tricks. E.g., particle acceleration can be used and in hot merger. 
Lithium reactions can greatly help starting and even maintaining the reaction.

The paper has also proposed another helper reaction, which also may be tested in the future: That between boron and hydrogen.

\section{Acknowledgement}

This text was acknowledged and appreciated by Dr. Veturia CHIROIU Honorific member of Technical Sciences Academy of Romania (ASTR) PhD.

\section{Funding Information}

Petrescu F.I., 2012, Cold nuclear fusion. Plasma Physics And Fusion Technology (S70), INIS 44(16)

\section{Author's Contributions}

All the authors contributed equally to prepare, develop and carry out this manuscript.

\section{Ethics}

Authors declare that are not ethical issues that may arise after the publication of this manuscript. This article is original and contains unpublished material.

\section{References}

Atzeni, S. and J. Meyer-ter-Vehn, 2004. Nuclear Fusion Reactions. In: The Physics of Inertial Fusion, University of Oxford Press, ISBN-13: 978-0-19-856264-1.

Avantages de la Fusion, La Science. ITER. https://www.iter.org/fr/sci/Fusion.

Brown, L.M. and H. Rechenberg, 1996. The Origin of the Concept of Nuclear Forces. 1st Edn., CRC Press, ISBN-10: 0750303735, pp: 392.

Coulomb, C.A., 1788. Premier mémoire sur l'électricité et le magnétisme. Histoire de l'Académie Royale des Sciences. Imprimerie Royale.

De Ninno, A., A. Frattolillo, A. Rizzo and E. Del Giudice, 2002. Preparata, experimental evidence of $4 \mathrm{He}$ production in a cold fusion experiment. ENEA Technical Report, RT2002/41/FUS.

Gerstner, E., 2009. Nuclear energy: The hybrid returns. Nature, 460: 25-28. DOI: 10.1038/460025a

Halliday, D. and R. Robert, 1966. Physics, Part II. 1st Edn., John Wiley and Sons, Inc., New York.

Harold Urey, from Wikipedia, the free encyclopedia. https://en.wikipedia.org/wiki/Harold_Urey.

Hoffman, M., 2013. Or how to make fusion power viable. What is the Lawson criteria. Science World Reports.
Hydrogen, from Wikipedia, the free encyclopedia. https://en.wikipedia.org/wiki/Hydrogen.

Jones, S.E., 1986. Muon-catalysed fusion revisited. Nature, 321: 127-133. DOI: 10.1038/321127a0

Krane, K.S., 1987. Introductory Nuclear Physics. 3rd Edn., Wiley and Sons, New York, ISBN-10: 047180553X, pp: 864.

Kramer, D., 2011. DOE looks again at inertial fusion as a potential clean-energy source. Phys. Today, 64: 26-26 DOI: $10.1063 / 1.3563814$

Miller, R.C. and R.A. Krakowski, 1984. Proc. MuonCatalysed Fusion Workshop.

Moses, E.I., R.N. Boyd, B.A. Remington, C.J. Keane and R. Al-Ayat, 2009. The national ignition facility: Ushering in a new age for high energy density science. Phys. Plasmas, 16: 041006-041006. DOI: $10.1063 / 1.3116505$

Palmer, D., 1997. Hydrogen in the universe. NASA.

Park, J., R.A. Nebel, S. Stange and S.K. Murali, 2005. Experimental observation of a periodically oscillating plasma sphere in a gridded inertial electrostatic confinement device. Phys. Rev. Lett., 95: 015003-015003.

DOI: 10.1103/PhysRevLett.95.015003

Petrescu, F.I., 2012. Cold nuclear fusion. Plasma Physics and Fusion Technology (S70), INIS.

Petrescu, F.I. and J.K. Calautit, 2016a. About nano fusion and dynamic fusion. Am. J. Applied Sci., 13: 261-266 DOI: 10.3844/ajassp.2016.261.266.

Petrescu, F.I. and J.K. Calautit, 2016b. About the light dimensions. Am. J. Applied Sci., 13: 321-325. DOI: 10.3844/ajassp.2016.321.325.

Petrescu, F.I. and R.V. Petrescu, 2012. News in Physics. 1 st Edn., Books on Demand, ISBN-13: 978-3848229642, pp: 82.

Petrescu, F.I., A. Apicella, R.V. Petrescu, S. Kozaitis and R. Bucinell et al., 2016. Environmental protection through nuclear energy. Am. J. Applied Sci., 13: 941-946.

DOI: 10.3844/ajassp.2016.941.946.

"Progress in Fusion". ITER. Retrieved from: http://www.iter.org/sci/beyonditer

Ragheb, M., 2013. Nuclear processes, the strong force. University of Illinois.

Reid, R.V., 1968. Local phenomenological nucleonnucleon potentials. Annals Phys., 50: 411-448.

Reynolds, S.P., K.J. Borkowski, D.A. Green, U. Hwang and I. Harrus et al., 2008. The youngest galactic supernova remnant: G1.9+0.3. Astrophys. J. Lett., 680: L41-L44.

Schewe, P. and B. Stein, 2005. Pyrofusion: A roomtemperature, palm-sized nuclear fusion device. Phys. News Update. 
Shultis, J.K. and R.E. Faw, 2002. Fundamentals of Nuclear Science and Engineering. 1st Edn., CRC Press, New York, ISBN-10: 0824708342, pp; 520.

Thermonuclear weapon, from Wikipedia, the free encyclopedia.

https://en.wikipedia.org/wiki/Thermonuclear_weapon.

Tipler, P. and R. Llewellyn, 2008. Modern Physics. 5th Edn., W. H. Freeman and Company, ISBN-13: 978-0-7167-7550-8.

Vermote, S., C. Wagemans, O. Serot and P. Geltenbort, 2008. Comparative study of the ternary particle emission in 243-Cm (nth,f) and 244-Cm(SF). Proceedings of the 6th International Conference on Dynamical Aspects of Nuclear Fission, World Scientific Publishing Co. Pte. Ltd., Singapore.

\section{Nomenclature}

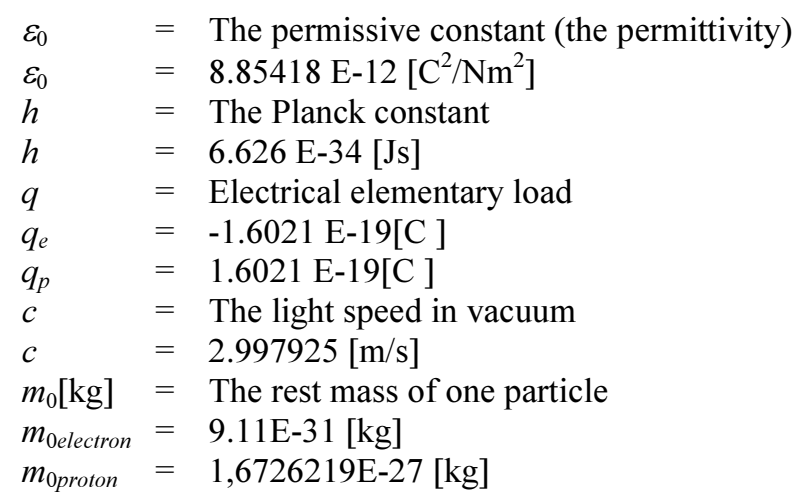

